\title{
MORFOMETRÍA DE LA CONCHA DE DONAX DENTICULATUS Y DONAX STRIATUS DE DOS PLAYAS DE CUBA ORIENTAL
}

\section{SHELL MORPHOMETRY OF DONAX STRIATUS AND DONAX DENTICULATUS FROM TWO BEACHES IN EASTERN CUBA}

\author{
Frank A. Ocaña ${ }^{1 *}$ y Alejandro Fernández ${ }^{1}$
}

\begin{abstract}
RESUMEN
Se han realizado estudios sobre morfología y ecología de Donax striatus y D. denticulatus en playas del Caribe, sin embargo, ninguna investigación ha abordado aspectos sobre morfometría en vistas de aplicar esta información para establecer pautas para la explotación de este recurso. Los objetivos de este trabajo son: cuantificar las relaciones entre las variables morfométricas, así como verificar si el Indicador de Estabilización de la Forma (IEF) puede ser útil para determinar la talla mínima de captura para estas dos especies. $D$. striatus alcanza mayor tamaño que $D$. denticulatus. En todos los casos, la correlación entre las variables morfométricas es altamente significativa. En $D$. striatus se puede discernir fácilmente que ocurre una estabilización de la forma a una longitud de $15.48 \mathrm{~mm}$, sin embargo, para $D$. denticulatus no se puede diferenciar con claridad un punto de inflexión en las curvas de los cocientes entre las medidas morfométricas. Los resultados no parecen ser concluyentes en cuanto a la utilización de IEF como una medida para proponer pautas de manejo para las pesquerías de las especies de Donax. Se sugiere que la longitud mínima de captura de $D$. striatus y $D$. denticulatus sea establecida en $20 \mathrm{~mm}$, con el fin de permitir que los individuos se hayan reproducido al menos una vez en su ciclo de vida.
\end{abstract}

Palabras claves: Bivalvia, Donax, playas arenosas, relaciones morfométricas, Cuba.

\begin{abstract}
Studies have been conducted on the morphology and ecology of Donax striatus and D. denticulatus in the Caribbean beaches. However, no investigation has been focused on their morphometry as to apply this information to determine a guideline for the exploitation of this resource. The objective of this paper is to quantify the relationships between morphometric variables and verify whether the Shell Shape Stabilization Indicator (IEF, Spanish acronym) is useful to determine the minimum size of catch for the two species. $D$. striatus is larger than $D$. denticulatus. In all cases, correlation between the morphometric variables is highly significant. In D. Striatus, shell shape stabilization is visible to occur at $15.48 \mathrm{~mm}$ in length; however, for $D$. denticulatus an inflexion point is not easily observed in the curves of the ratios between the morphometric measures. Results are not conclusive to propose the use of IEF as a guideline for fishery of Donax species. The minimum size of catch of $D$. striatus and $D$. denticulatus suggested should be established at $20 \mathrm{~mm}$ to allow individuals to reproduce at least once during their life cycle.
\end{abstract}

Keywords: Bivalvia, Donax, sandy beaches, morphometric relationships, Cuba.

1 Centro de Inv. y Serv. Amb. y Tec. 18 s/n. esq. a Maceo. Rpto. "El Llano”. Holguín, Cuba. *franko@cisat.cu

Recibido 9-II-2011

Aceptado 15-V-2011

DOI: http://dx.doi.org/10.15359/revmar.3.5 


\section{INTRODUCCIÓN}

Los moluscos bivalvos del género Donax son los organismos dominantes en las playas arenosas de las zonas tropicales y templadas (Ansell, 1983). Estas almejas poseen forma de cuña, lo que parece ser una adaptación que les favorece migrar en la zona intermareal y un rápido enterramiento en el sedimento (Stanley, 1970). Además del papel ecológico que desempeñan estos organismos en las playas, las poblaciones de Donax tienen un elevado valor para la pesca comercial y la recreativa en muchos países (McLachlan et al. 1996), por lo que una correcta evaluación de las características de estas especies y sus parámetros poblacionales es un elemento imprescindible para su explotación. En este sentido, Gil et al. (2007) proponen un método simple para inferir acerca de la longitud de la concha en que los individuos maduran sexualmente pudiendo ser recolectados después de esta talla, asegurando que se hayan logrado reproducir al menos una vez.

En Cuba, el género Donax está representado por dos especies, $D$. denticulatus y D. striatus, las cuales, según Wade (1967a), presentan el mismo rango de distribución. Sobre estas especies se han realizado estudios de su ecología en playas del Caribe (García et al. 2003; Marcano et al. 2003; Ocaña et al. 2010), sin embargo, ninguna investigación ha abordado aspectos sobre morfometría en vistas de aplicar esta información para establecer pautas para la explotación de este recurso. Los objetivos de este trabajo son: cuantificar las relaciones de la forma de la concha en $D$. denticulatus y $D$. striatus y determinar la longitud en que los organismos alcanzan su forma definitiva como una medida que establezca las tallas mínimas de captura para una explotación de este recurso.

\section{MATERIALES Y MÉTODOS}

Se tomaron muestras en una población de $D$. denticulatus en playa Carenero, Niquero, Granma, que no está siendo utilizada por una pesquería artesanal y de una población de D. striatus en playa Las Balsas, Gibara, Holguín, que está siendo explotada artesanalmente por los pobladores locales (Fig. 1). El muestreo se realizó mensualmente desde febrero hasta julio de 2008, durante marea baja.

En cada playa se seleccionaron tres estaciones fijas. La obtención de las muestras se realizó empleando un cilindro de PVC, con un diámetro de $18 \mathrm{~cm}$ y una altura de $30 \mathrm{~cm}$, introduciéndolo en el sustrato arenoso hasta una profundidad de 20 cm (unidad de muestreo, UM). En cada estación se tomaron muestras en estratos separados a intervalos de $1 \mathrm{~m}$ perpendicular a la línea de costa, a partir del punto de inflexión o escalón de la playa en la zona intermareal hasta el límite superior de la zona de barrido del oleaje. En cada estrato se extrajeron tres cilindros de $0.025 \mathrm{~m}^{2}$, separados cada $1 \mathrm{~m}$.

El sedimento fue cribado en un tamiz de $1 \mathrm{~mm}$ de abertura de malla. Los individuos recolectados se introdujeron en bolsas plásticas y se congelaron. En el laboratorio, a cada individuo se le determinaron las medidas de los tres ejes (largo: máxima distancia del eje antero-posterior; alto: máxima distancia del eje ventrodorsal a través de la mitad de la concha; y ancho: máxima distancia del eje lateral entre las dos valvas de la concha cerrada), con el empleo de un Vernier digital, con una precisión de $0.01 \mathrm{~mm}$.

El establecimiento de estas relaciones morfométricas se realizó mediante la ecuación general $\mathrm{Y}=\mathrm{a}+\mathrm{b} * \mathrm{X}$, adaptada a las variables analizadas de la forma siguiente: 


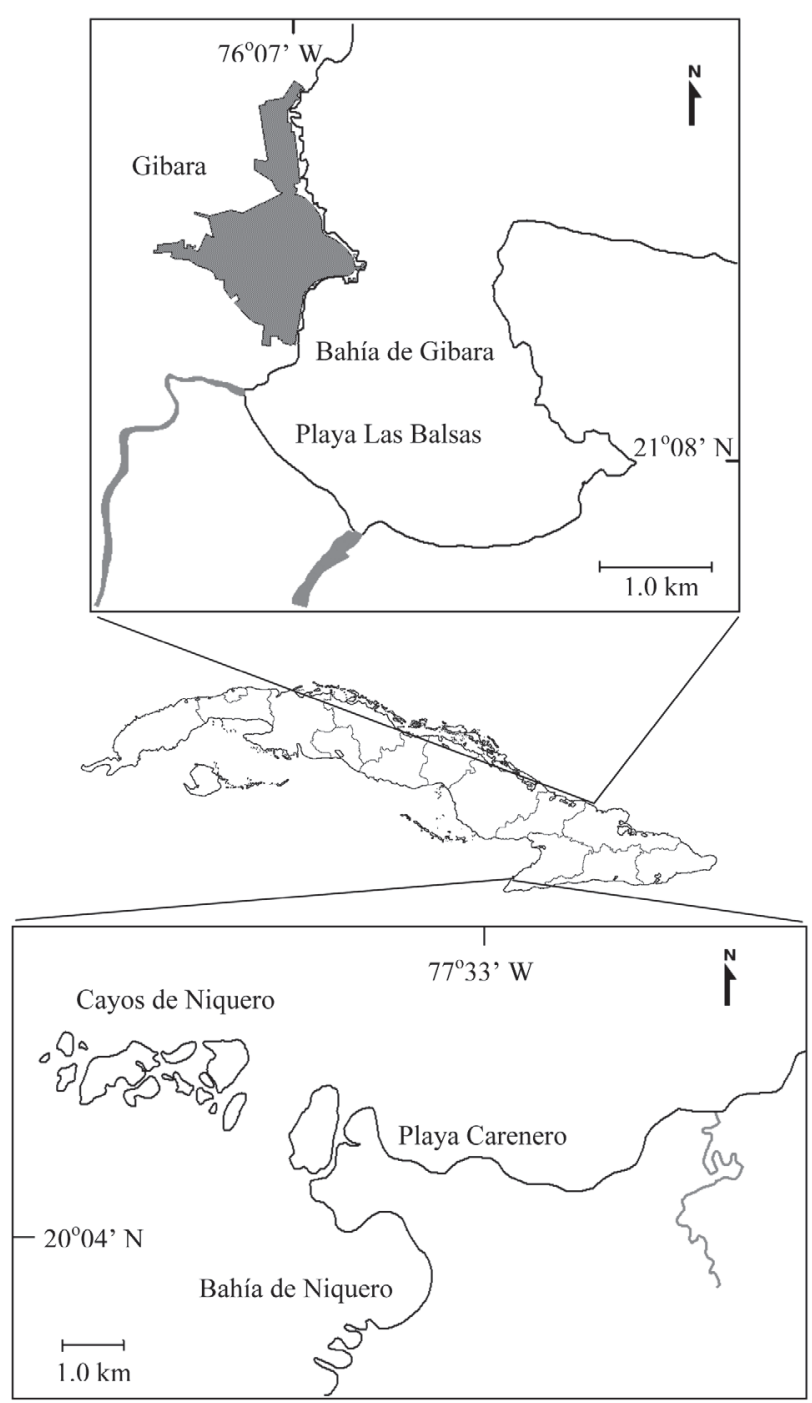

Fig. 1. Ubicación geográfica de las playas Las Balsas y Carenero

Fig. 1. Geographical location of Las Balsas and Carenero beach

$\mathrm{Al}=\mathrm{a}+\mathrm{b} * \mathrm{~L} \quad \mathrm{An}=\mathrm{a}+\mathrm{b} * \mathrm{~L} \quad \mathrm{An}=\mathrm{a}+\mathrm{b} * \mathrm{Al} \quad 1 \mathrm{o}$ es alométrico negativo o positivo cuando $\mathrm{b}<1$ o $\mathrm{b}>1$, respectivamente.

Donde: L - largo; Al - alto; An - ancho; Los parámetros a y $b$ fueron estimados a - intersecto (coeficiente de crecimiento inicial); y b - pendiente de la recta (coeficiente de crecimiento relativo). El coeficiente de alometría es expresado por el parámetro b de la ecuación de regresión lineal, indicando que el crecimiento es isométrico cuando $b=$ por análisis de regresión lineal con los datos transformados a logaritmo y el grado de asociación entre las variables fue calculado por el coeficiente de determinación $\left(r^{2}\right)$. Las diferencias estadísticas fueron consideradas con un nivel de significación de 95\% ( $\alpha=$ 0.05) (Zar, 1999) para todos los parámetros. 
Para verificar si los valores de b resultantes de las tres ecuaciones son significativamente diferentes del valor isométrico $(\mathrm{b}=1)$, fue aplicada una prueba $t$ (Ho: $\mathrm{b}$ $=1$ ), con un intervalo de confianza de \pm 95\% ( $\alpha=0.05)$ (Sokal y Rohlf, 1995; Zar, 1999; Gaspar et al. 2002).

Para determinar la talla a la que los organismos alcanzan su forma definitiva se empleó el método propuesto por Gil et al. (2007), que denominan Indicador de Estabilización de la Forma (IEF). Este método es muy simple y consiste en calcular las razones entre las tres medidas lineales de la concha (largo, alto y ancho). Para ello se separan los individuos en intervalos de clases de $1 \mathrm{~mm}$ y se calcula la media del largo, alto y ancho para cada intervalo. Las razones que se calculan son las siguientes: An/L, Al/L y Al/An. Las razones se presentan en porcentajes.

\section{RESULTADOS}

Los resultados obtenidos de las relaciones biométricas son presentados en el cuadro 1 . Se observa que D. striatus alcanza mayor tamaño que $D$. denticulatus y en todos los casos la correlación entre las variables morfométricas es altamente significativa. En las poblaciones analizadas de ambas especies, las relaciones entre el ancho y el largo, así como entre el ancho y el alto, son positivas, sin embargo, en $D$. denticulatus se observa una tendencia hacia la isometría. En el caso de la relación entre el alto y el largo existe un crecimiento relativo isométrico en $D$. denticulatus y alométrico negativo en $D$. striatus con tendencia a la isometría. Esto se traduce en que para ambas especies la tasa de crecimiento en ancho es mayor que la tasa de crecimiento en largo y alto, indicando que estos organismos tienen conchas globosas.
Los cálculos de las razones entre las diferentes dimensiones de la concha se realizaron con el fin de determinar el punto de inflexión de la recta resultante de las regresiones de las relaciones alométricas en las dos especies de Donax. Según Wilbur y Owen (1964), los cambios en la razón de las dimensiones de la concha expresan alteraciones del patrón en los estadios de crecimiento, siendo expresiones del punto de inflexión de la recta resultante del análisis de alometría. A partir de este punto de inflexión se supone que ya ha ocurrido la estabilización del crecimiento, a lo que Gil et al. (2007) denominan IEF.

En las figuras 2 y 3 se observan las curvas resultantes del cálculo de las razones entre las variables morfométricas analizadas. En ambos casos existe una estrecha correspondencia con los resultados de las ecuaciones biométricas. En $D$. striatus se puede discernir fácilmente que ocurre una estabilización de la forma a una longitud de $15.48 \mathrm{~mm}$, teniendo en cuenta las curvas de las relaciones entre ancho/largo y ancho/alto, que en la ecuación biométrica presentan valores que difieren del valor isométrico $(b=1)$. Sin embargo, analizando las curvas para $D$. denticulatus no se puede diferenciar claramente un punto de inflexión, lo que coincide con los valores de las ecuaciones biométricas calculadas para esta especie.

\section{DISCUSIÓN}

Entre todos los bivalvos, los de playas arenosas son los enterradores más rápidos (Stanley, 1970; McLachlan et al. 1995). Los donácidos parecen haber evolucionado hacia formas más acuñadas, tal vez como una adaptación que incrementa la velocidad de enterramiento, la estabilidad y la orientación ante la resaca del 
Cuadro 1. Estadística descriptiva de las muestras y relaciones biométricas de Donax striatus y D. denticulatus de dos playas de Cuba oriental (N: tamaño de muestra, L: longitud, D.E.: desviación estándar, E.S.: error estándar, b: coeficiente de crecimiento relativo) Table 1. Sample descriptive statistics and biometric relationships of Donax striatus and D. denticulatus from two beaches on eastern Cuba (N: sample size, L: length, D.E: standard deviation, E.S.: standard error, b: relative growth rate)

\begin{tabular}{lcllcccll}
\hline Especie & N & $\begin{array}{l}\text { L media } \pm \text { D.E. } \\
\text { (min. - máx.) }\end{array}$ & $\begin{array}{l}\text { Relación } \\
\text { biométrica }\end{array}$ & Ecuación biométrica & $\begin{array}{l}\text { Coeficiente de } \\
\text { determinación }\left(r^{2}\right)\end{array}$ & $\begin{array}{c}\text { E.S. de b } \\
\text { Valor } \\
\text { de t }\end{array}$ & $\begin{array}{l}\text { Relación } \\
\text { alométrica }\end{array}$ \\
\hline D. striatus & 282 & $13.11 \pm 5.26$ & $\mathrm{Al} / \mathrm{L}$ & $\mathrm{Al}=-0.182+0.988 * \mathrm{~L}$ & 0.993 & 0.005 & -2.4 & negativo \\
& & $(3.48-28.24)$ & $\mathrm{An} / \mathrm{L}$ & $\mathrm{An}=-0.549+1.133 * \mathrm{~L}$ & 0.981 & 0.009 & 14.8 & positivo \\
& & $\mathrm{An} / \mathrm{Al}$ & $\mathrm{An}=-0.340+1.146 * \mathrm{Al}$ & 0.988 & 0.007 & 20.86 & positivo \\
& & & $\mathrm{Al} / \mathrm{L}$ & $\mathrm{Al}=-0.178+1.005 * \mathrm{~L}$ & 0.997 & 0.003 & 1.67 & isométrico \\
D. denticulatus & 285 & $15.62 \pm 5.77$ & $\mathrm{An} / \mathrm{L}$ & $\mathrm{An}=-0.435+1.028 * \mathrm{~L}$ & 0.989 & 0.006 & 4.7 & positivo \\
& \multirow{2}{*}{$3.94-24.39)$} & & $\mathrm{An} / \mathrm{Al}$ & $\mathrm{An}=-0.252+1.022 * \mathrm{Al}$ & 0.991 & 0.006 & 3.67 & positivo \\
\hline
\end{tabular}

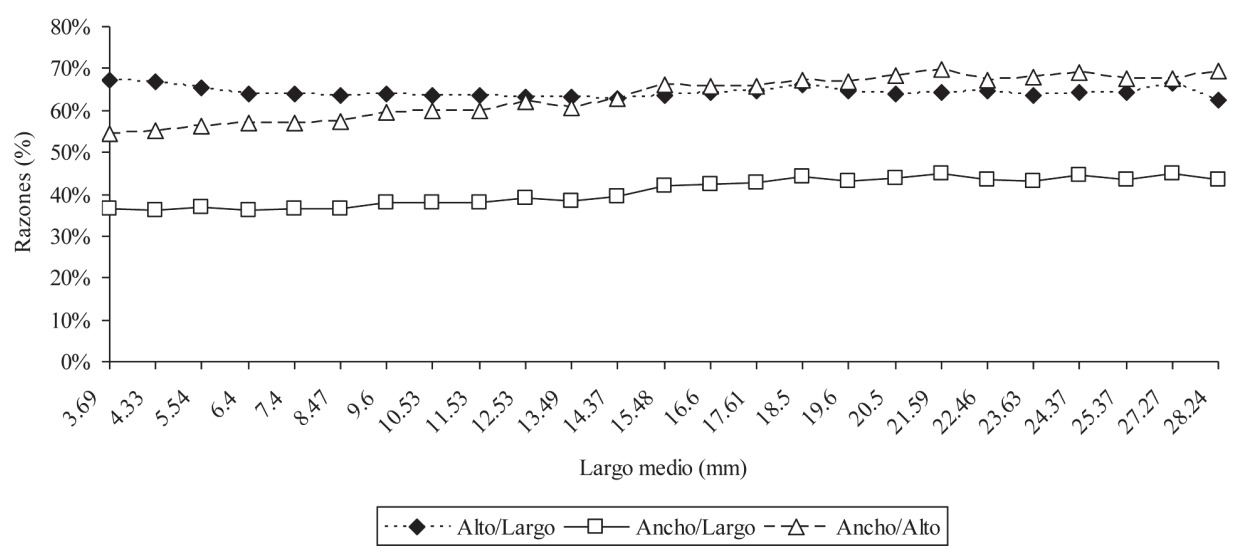

Fig. 2. Gráfico de las razones entre las dimensiones de la concha de Donax striatus

Fig. 2. Graphic of the ratios between shell dimensions of Donax striatus

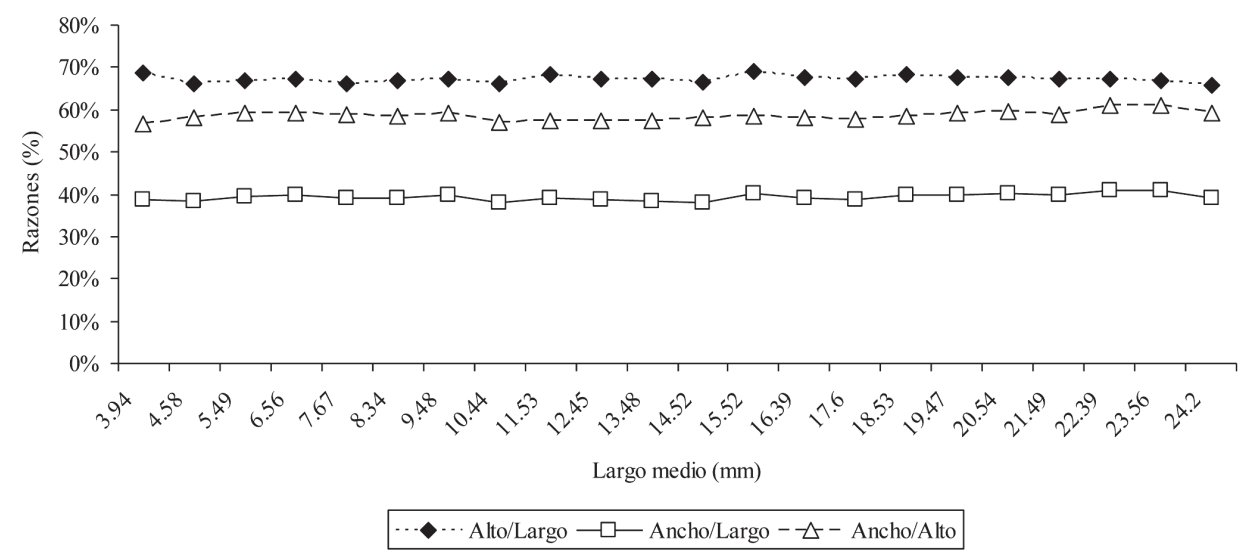

Fig. 3. Gráfico de las razones entre las dimensiones de la concha de Donax denticulatus

Fig. 3. Graphic of the ratios between shell dimensions of Donax denticulatus 
Cuadro 2. Comparación de relaciones alométricas entre diferentes especies del género Donax Table 2. Comparison of allometric relationships among different Donax species

\begin{tabular}{llccc}
\hline \multicolumn{1}{c}{ Especie } & \multicolumn{1}{c}{ Referencia } & \multicolumn{3}{c}{ Relaciones alométricas } \\
\cline { 2 - 4 } & & Alto/Largo & Ancho/Largo & Ancho/Alto \\
\hline D. trunculus & Salas-Casanova (1987) & 0.49 & & \\
& Bayed (1998) & 1.17 & 0.98 & 0.83 \\
& Gaspar et al. (2002) & $0.88-0.90$ & $1.00-1.12$ & \\
D. vittatus & Salas-Casanova (1987) & 0.50 & & \\
D. semistriatus & Salas-Casanova (1987) & 0.51 & & \\
D. venustus & Salas-Casanova (1987) & 0.48 & & \\
D. serra & Soares et al. (1998) & $0.68-0.71$ & $0.39-0.44$ & \\
D. striatus & Wade (1967b) & $0.35-0.38$ & & \\
D. incarnatus & Thippeswamy y Joseph & 0.67 & 0.38 & \\
& (1992) & & & 0.91 \\
D. variabilis & Nelson et al. (1993) & 0.51 & 0.58 & 1.10 \\
D. parvula & Nelson et al. (1993) & 0.62 & 0.53 & 1.38 \\
D. hanleyanus & Gil y Thomé (2000) & 0.92 & 1.29 & \\
\hline
\end{tabular}

oleaje. En los estudios realizados sobre la morfometría de la concha en especies del género Donax, se observan diferencias en las relaciones alométricas; en el cuadro 2 se puede constatar que una misma especie en diferentes localidades puede tener distintos valores de su coeficiente de alometría. Gaspar et al. (2002) encontraron estas diferencias incluso en los individuos de $D$. trunculus que se localizaban en diferentes profundidades. Wade (1967b) encontró diferencias en el coeficiente de alometría entre dos poblaciones de $D$. striatus, atribuyendo este fenómeno a diferencias en la densidad de individuos.

De forma general, las relaciones entre el alto y el largo presentan una alometría negativa en todas las especies, a excepción de D. trunculus en el litoral de Marruecos (Bayed, 1998) y D. denticulatus en playa Carenero. Según Gaspar et al. (2002), la forma estrecha y alargada de la concha es una estrategia adaptativa que favorece la eficiencia del enterramiento. En cuanto a la relación entre el ancho con el largo y el alto no se observa un patrón definido para las especies. Wade (1968), al comparar dos poblaciones de $D$. denticulatus en Jamaica, constató diferencias en las tasas de crecimiento y en el tamaño máximo de los individuos y justificó tales diferencias en función de la disponibilidad de alimento, por tanto, los factores ambientales podrían ser la causa de tales diferencias entre poblaciones de la misma especie.

La expresión gráfica de las relaciones entre los cocientes de las variables morfométricas refleja los resultados obtenidos en el cálculo de las alometrías. Las curvas resultantes de la relación alto/largo para las dos especies no muestran claramente un punto de inflexión, por lo que no brindan suficiente información para determinar un cambio en la tasa de crecimiento. Las curvas referidas a las relaciones ancho/largo y ancho/alto son las que mejor reflejan la presencia de un punto de inflexión a los $15.48 \mathrm{~mm}$ para $D$. striatus, sin embargo, en $D$. denticulatus estas dos 
rectas se comportan prácticamente estables sin presentar un punto de inflexión.

Siguiendo los criterios de Gil et al. (2007) se puede considerar que la población de D. striatus en playa Las Balsas alcanza su forma definitiva entre 15 y 16 $\mathrm{mm}$, pero en $D$. denticulatus no se evidencia un cambio en la recta, lo que puede conllevar a falsas interpretaciones. Si el Indicador de Estabilización de la Forma de la concha puede ser una medida para determinar la talla de primera maduración, entonces en $D$. striatus los individuos comienzan a ser adultos a partir de los 15 mm. Gil y Thomé (2000) encontraron que la concha de $D$. hanleyanus, perteneciente a una población del sur de Brasil, alcanzó su forma definitiva a los $12 \mathrm{~mm}$; posteriormente a esta misma población se le determinó, mediante análisis histológicos, que su talla de primera maduración estuvo comprendida entre los 12 y 13 mm (Gil y Thomé, 2004).

A pesar de no haber diferenciación en las curvas de las razones entre las medidas morfométricas en $D$. denticulatus, también se podría tomar la longitud de $15 \mathrm{~mm}$ como talla de primera maduración, debido a que estas curvas son relativamente similares a las expresadas en la figura 2 para D. striatus.

\section{CONCLUSIONES}

Las variables morfométricas analizadas para las especies $D$. striatus y $D$. denticulatus presentan una alta correlación, siendo mayor la tasa de crecimiento en ancho que en largo y alto. Las curvas resultantes de las razones entre ancho/largo $\mathrm{y}$ ancho/alto son las que mejor explican la estabilización en el crecimiento. Para D. striatus, el crecimiento se estabiliza a una longitud media de $15.48 \mathrm{~mm}$, sin embargo, para $D$. denticulatus no se puede discernir claramente la longitud media de estabilización de la forma de la concha. Los resultados presentados no parecen ser concluyentes en cuanto a la utilización de IEF como una medida para proponer pautas de manejo para las pesquerías de las especies de Donax; para ello sería necesario realizar una serie de análisis histológicos en diferentes poblaciones para determinar la talla de primera maduración y, posteriormente, relacionar los resultados de los cocientes de las diferentes variables morfométricas. Analizando todos estos aspectos en conjunto y teniendo en cuenta un enfoque precautorio en el uso de los recursos, se puede sugerir que la longitud mínima de captura de $D$. striatus y $D$. denticulatus sea establecida en $20 \mathrm{~mm}$, con el fin de permitir que los individuos se hayan reproducido al menos una vez.

\section{AGRADECIMIENTOS}

Agradecemos la colaboración de Alexis Silva, Yanet Apín, Vladimir Martínez y Yuself Cala en la toma de muestras. A Marko Herrmann por facilitarnos gran parte de la literatura.

\section{BIBLIOGRAFÍA}

Ansell, A. D. (1983). The biology of the genus Donax. En A. McLachlan, T. Erasmus \& W. Junk (Eds.), Developments in hydrobiology, 19. Sandy beaches as ecosystems (pp. 607-635). The Hague, The Netherlands: Dr. W. Junk Publishers.

Bayed, A. (1998). Variabilité de la croissance de Donax trunculus sur le littoral marocain. En J. Lleonart (Ed.), Dynamique des populations 
marines $=$ Marine populations dynamics (pp. 11-23). Zaragoza, España: CIHEAM-IAMZ.

García, N., Prieto, A., Alzola, R. \& Lodeiros, C. (2003). Crecimiento y distribución de tallas de Donax denticulatus (Mollusca: Donacidae) en playa Brava, península de Araya, estado Sucre, Venezuela. Rev. Cient. LUZ, 13(6), 464-470.

Gaspar, M. B., Chícharo, L. M., Vasconcelos, P., García, A., Santos, A. R. \& Monteiro, C. C. (2002). Depth segregation phenomenon in Donax trunculus (Bivalvia: Donacidae) populations of the Algarve coast (southern Portugal). Sci. Mar., 66(2), 111-121.

Gil, G. M. \& Thomé, J. W. (2000). Morfometria da concha em Donax hanleyanus Philippi, 1847 (Mollusca, Bivalvia, Donacidae) e sua relação com as zonas de praia. A. Biol. Leop., 22(2), 161-170.

Gil, G. M. \& Thomé, J. W. (2004). Proporção sexual e comprimento de concha na primeira maturação sexual em Donax hanleyanus Philippi (Bivalvia, Donacidae) no Rio Grande do Sul, Brasil. Rev. Bras. Zool., 21(2), 345-350.

Gil, G. M., Troncoso, J. S \& Thomé, J. W. (2007). Manual para Manejo e Otimização da Exploração Comercial de Moluscos Bivalves. Porto Alegre, Brasil: Edição do autor.

Marcano, J. S., Prieto, A., Lárez, A. \& Salazar, H. (2003). Crecimiento de Donax denticulatus (Linné, 1758) (Bivalvia: Donacidae) en la ensenada La Guardia, isla de Margarita, Venezuela. Zoot. Trop., 21(3), 237-259.

McLachlan, A., Dugan, J. E., Defeo, O., Ansell, A. D., Hubbard, D. M., Jarami1lo, E. \& Penchaszadeh, P. E. (1996).
Beach clam fisheries. Oceanogr. Mar. Biol. Ann. Rev., 34, 163-232.

McLachlan, A., Jaramillo, E., Defeo, O., Dugan, J. E., de Ruyck, A. \& Coetzee, P. (1995). Adaptations of bivalves to different beach types. J. Exp. Mar. Biol. Ecol., 187, 147-160.

Nelson, W. G., Bonsdorff, E. \& Adamkewics, L. (1993). Ecological, morphological, and genetics differences between the sympatric bivalves Donax variabilis Say, 1822, and Donax parvula Philippi, 1849. The Veliger, 36(4), 317-322.

Ocaña, F. A., Fernández, A., Silva, A., González, P. A. \& García, Y. (2010). Estructura poblacional de Donax striatus (Bivalvia, Donacidae) en playa Las Balsas, Gibara, Cuba. Rev. Mar. Cost., 2, 27-38.

Salas-Casanova, C. (1987). The Donacidae of the Bay of Malaga (Spain). Taxonomy. Basteria, 51(1-3), 33-50.

Soares, A. G., Callahan, R. K. \& de Ruyck, A. M. C. (1998). Microevolution and phenotypic plasticity in Donax serra Röding (Bivalvia: Donacidae) on high energy sandy beaches. J. Mollus. Stud., 64, 407-421.

Sokal, R. R. \& Rohlf, F. J. (1995). Biometry: the principles an practice of Statistics in Biological Research. New York, EE.UU.: W. H. Freeman and Company.

Stanley, S. M. (1970). Relation of shell form to life habits in the Bivalvia (Mollusca). Mem. Geol. Soc. Amer., 125, 1-296.

Thippeswamy, S. \& Joseph, M. M. (1992). Allometry in the wedge clam, Donax incarnatus (Gmelin) from Panambur beach, Mangalore. Ind. J. Mar. Sci., 21, 161-163. 
Wade, B. A. (1967a). Studies on the biology of the West Indian beach clam Donax denticulatus Linne. Ecology. Bull. Mar. Sci., 17, 149-174.

Wade, B. A. (1967b). On taxonomy, morphology, and ecology of the beach clam, Donax striatus Linne. Bull. Mar. Sci., 17, 723-740.

Wade, B. A. (1968). Studies on the biology of the West Indian beach clam,
Donax denticulatus. Linne. Life History. Bull. Mar. Sci., 18, 876-901.

Wilbur, K. M. \& Owen, G. (1964). Growth. En K. M. Wilbur \& C. M. Yonge (Eds.), Physiology of Mollus$c a$ (pp. 211-242). New York, EE.UU.: Academic Press.

Zar, J. H. (1999). Biostatistical Analysis. (4th. ed.). New Jersey, EE.UU.: Prentice-Hall Inc. 